\title{
Using Single-Beam Optical Tweezers for the Passive Microrheology of Complex Fluids
}

\author{
Iliya D. Stoev*, Alessio Caciagli ${ }^{+}$, Zhongyang Xing ${ }^{+}$, Erika Eiser ${ }^{*}$ \\ Department of Physics, University of Cambridge \\ Cavendish Laboratory, 19 JJ Thomson Avenue, Cambridge CB3 0HE, United Kingdom
}

\begin{abstract}
One important aspect of the complete physical characterization of novel viscoelastic materials is the assessment of their response on short timescales. Optical tweezers, equipped with a fast quadrant photodiode, aid in fulfilling this task by providing high-frequency viscoelastic information about the sample. In passive microrheology, this is normally achieved by extracting rheological information from the thermal motion of an optically trapped bead embedded in a test fluid. Here we present the calibration and use of optical tweezers to study the formation of thermally reversible DNA hydrogels. We complement our results with rheological data from dynamic light scattering, video microscopy and conventional bulk rheology. Merging experimental data from different techniques allows us to study the viscoelastic behavior of these DNA networks over a wide frequency-band and the scaling of the complex viscoelastic modulus at the two frequency extremes. By analyzing the high-frequency behavior of our transient network, we prove the semi-flexible polymer nature of DNA and provide an estimate of its persistence length.
\end{abstract}

Keywords: optical trapping, laser tweezers, passive microrheology, light scattering, viscoelasticity, DNA hydrogel

\section{INTRODUCTION}

Ever since the pioneering work of Ashkin et al., ${ }^{1}$ optical tweezers (OT) have found wide use in studying the mechanics of biological systems. Confining micron-sized colloids inside a harmonic potential is a key feature of optical trapping. Block et al. $^{2}$ used this characteristic of single-beam OT to show the intermittent movement of a kinesin protein along microtubules. In another biological assay, Wang and co-workers ${ }^{3}$ demonstrated the stretching of single-stranded (ss) DNA by optically manipulating a dielectric bead attached to one end of the molecule. Micromanipulation using light proved its effectiveness and hence became an indispensable tool for probing the internal structure of new materials.

The term "microrheology" encompasses many techniques, all of which involve analyzing the trajectory of a particle in order to extract rheological information about its surrounding medium. Common ways of obtaining and analyzing this trajectory are video recording by a high-speed camera ${ }^{4}$ or back focal plane (BFP) interferometry using a quadrant photodiode (QPD). ${ }^{5}$ These two methods yield consistent data ${ }^{6}$ and represent a form of passive microrheology (MR), where the thermal motion of an embedded particle yields a time-fluctuating positional signal. Data conversion then produces the bead's mean-squared displacement (MSD) and position-autocorrelation function (PACF). This conversion allows calculating the complex viscoelastic modulus in Laplace space via the generalized Stokes-Einstein relation (GSER): ${ }^{7}$

$$
\tilde{G}(s)=\frac{k_{B} T}{\pi a s\left\langle\Delta \tilde{r}^{2}(s)\right\rangle},
$$

where $k_{B} T$ is the thermal energy, $a$ stands for the radius of the probe-bead and the term in pointed brackets represents the Laplace transform of the MSD at a frequency $s$. Equation (1) is a direct consequence of the fluctuation-dissipation theorem, where the velocity-autocorrelation function of a bead obeys the overdamped Langevin equation. Directly calculating the Laplace transform of a positional signal presents difficulties as it tends to induce large artefacts when applied to a finite non-periodic signal. This prompted the search for an alternative approach to the data analysis. Evans $e t$ $a l{ }^{8}$ proposed an elegant method by converting the MSD directly from real to Fourier space. In computational terms, this method had the advantage of using optimized FFT routines, which circumvented the need for direct integration known to cause substantial truncation errors. These errors became more apparent in the elastic response of the measured system. 
Evans and co-workers proposed oversampling as a possible way of overcoming the limitations associated with a finite sampling rate, where the latter comprised artefacts originating from the interpolation scheme and the Nyquist limit. Therefore, we chose Evans' method in the analysis section of this manuscript.

Unlike active MR, involving oscillatory driving of a probe-bead, passive MR merely tracks the path of the bead as it diffuses through the system of interest. While non-perturbing, this approach has its limitation since the signal-to-noise ratio is much lower in stiff samples. Nevertheless, due to its high sensitivity, passive MR can provide valuable rheological information about biological systems that exhibit non-linear frequency scaling at low strains. ${ }^{9}$

In order to facilitate the characterization of complex fluids, we constructed a single-beam OT system, equipped with fast detection of the movement of a trapped bead. The absence of diffractive optics in this simplified setup does not allow the generation of multiple or time-shared traps. This is normally performed through the use of acousto-optic deflectors ${ }^{10}$ or spatial light modulators. ${ }^{11}$ Such advanced optical components enable generating an arbitrary laser beam profile, thus allowing spinning and sorting of particles as well as measuring cross-correlations in particle diffusion. However, standard OT, equipped with a temperature control and a fast position detector, make for a powerful rheological tool. That tool is capable of complementing existing experimental techniques, such as conventional bulk rheology (BR) and lightscattering MR. While the latter can provide viscoelastic information over a broad frequency range, it only does so by estimating ensemble-averaged properties of the material. Therefore, any heterogeneities present in the sample remain untested explicitly. The small sample volume and the detection of a localized material response are among the strongest features of optical trapping using microscopic probes.

In this paper, we first introduce the hydrogel formed by DNA nanostars and the different equipment and methods used in our measurements (Section 2). This is followed by information about the calibration of our newly constructed OT and data analysis (Section 3). Finally, we present our results obtained for the DNA hydrogel with four different techniques for extracting its viscoelastic properties and comment on our findings (Section 4). In particular, we show that the highfrequency MR data reveal characteristic relaxation times on nanoscale.

\section{EXPERIMENTAL DETAILS}

\subsection{Materials}

We used two sample materials to demonstrate the suitability of four different experimental techniques for obtaining rheological parameters. Deionized water (purified by EMD Millipore Direct-Q, Fisher Scientific) was used in the calibration and testing of the newly constructed OT setup. The suitability of our setup was then tested by studying a DNA hydrogel sample consisting of two types of trivalent DNA nanostars (here referred to as Y- and Y'-shapes) with complementary ss DNA binding ends. Details of the full DNA single strands (SS 1-6), forming the Y- and Y'-shapes, are given in Table 1. The three SS 1-3 (or SS 4-6) were partially complementary, such that a core of the Y-shape (or Y'shape) formed three double-stranded (ds) DNA arms (shown in green). In addition, the 7-base-pair long ss "sticky ends" of the Y-shapes (blue) and Y'-shapes (red) were chosen to be complementary, such that when brought to below their melting temperature, they started to bind to each other forming a percolating, viscoelastic network. The flexibility of this network is provided by the four thymine bases (black) separating the core from the sticky end. A detailed discussion of the melting behavior of the cores can be found in the work of Xing et al. ${ }^{12}$

Table 1. Single-stranded DNA base sequences to form Y-and Y'-shapes in each one of the DNA strands: sticky ends (Y: blue, Y': red), rigid core (Y and $\mathrm{Y}^{\prime}$ : green) and non-binding flexible linkers made of thymines (black).

\begin{tabular}{|ll|}
\hline & DNA Sequences \\
SS 1 & 5'-ATC ACA GTt TTT GGA TCC GCA TGA TCC ATT CGC CGT AAG TA-3' \\
SS 2 & 5'-ATC ACA GTT TTT ACT TAC GGC GAA TGA CAC CGA ATC AGC CT-3' \\
SS 3 & 5'-ATC ACA GTT TTA GGC TGA TTC GGT GTG ATC ATG CGG ATC CA-3' \\
SS 4 & 5'-CTG TGA TTT TTT GGA TCC GCA TGA TCC ATT CGC CGT AAG TA-3' \\
SS 5 & 5'-CTG TGA TTT TTT ACT TAC GGC GAA TGA CAC CGA ATC AGC CT-3' \\
SS 6 & 5'-CTG TGA TTT TTA GGC TGA TTC GGT GTG ATC ATG CGG ATC CA-3' \\
\hline
\end{tabular}


All ss DNA was purchased from Integrated DNA Technologies (IDT) and then hybridized into nanostars using Cary 300 UV-Vis spectrophotometer (Agilent). The absorption at $260 \mathrm{~nm}$ was measured by ramping the temperature between 30 $80^{\circ} \mathrm{C}$ (nanostars) and $0-50^{\circ} \mathrm{C}$ (sticky ends). The concentration of each ss DNA used in the rheology experiments was 200 $\mu \mathrm{M}$ in $1 \mathrm{x}$ Tris-EDTA (TE) buffer (Sigma-Aldrich) with $\mathrm{pH} 8$, giving a final mass concentration of $2.5 \% \mathrm{w}$, corresponding to a concentration, at which a fully percolating gel can form. ${ }^{12}$

\subsection{Optical trapping}

Our homebuilt OT system is based on a Thorlabs model OTKB/M equipped with an infrared laser diode (975 $\mathrm{nm}$ wavelength and $140 \mathrm{~mW}$ maximum trapping power). The arrangement of the components and the trapping process are presented in Figure 1.

(a)

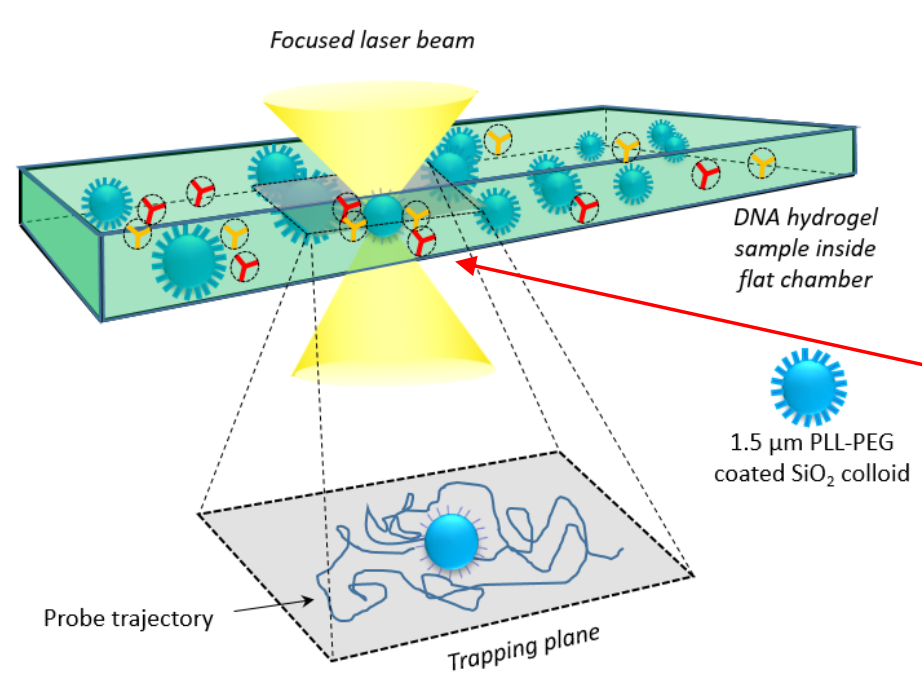

(b)

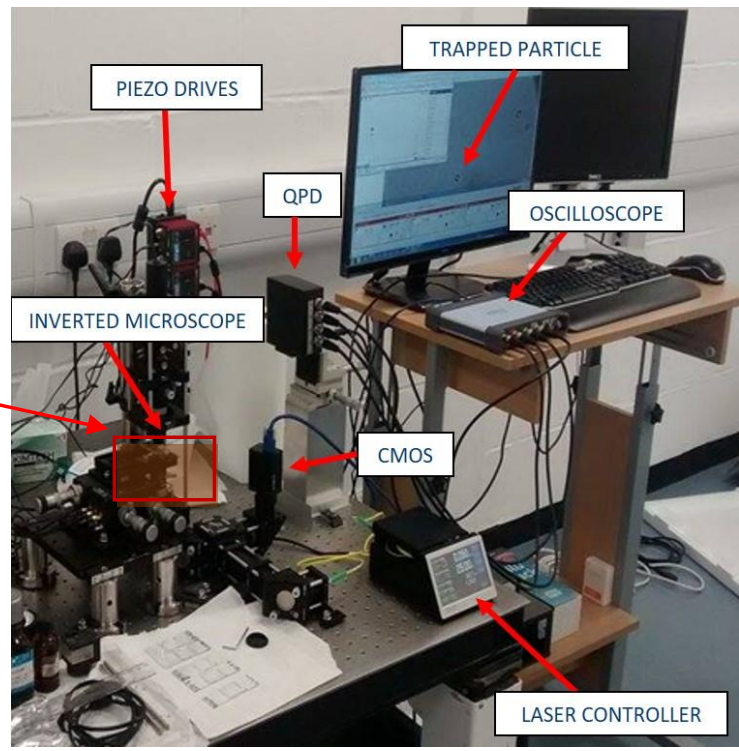

Figure 1. (a) Schematic representation of the OT trapping a silica probe-particle inside the DNA hydrogel sample, and its trajectory in time. (b) Photograph of the corresponding experimental setup and optical components used (enclosure not shown).

We modified the base OTKB/M setup in the following way: the trajectory of the probe-bead was monitored both by a CMOS camera and via BFP interferometry using an InGaAs QPD (Hamamatsu, model G6849-01). The analog-to-digital signal conversion was carried out using a picoscope (Pico Technology, model 5443B). To facilitate temperaturecontrolled measurements, we replaced the NanoMax stage from Thorlabs with a copper one. Furthermore, the hole in the center of the sample holder, which allows light to be transmitted, was covered by a sapphire window to minimize any heat loss. A Peltier unit, connected to a temperature controller, was fitted between the top of the copper stage and the cooling head of a fan (Corsair Components) that extracted any excess heat. We enclosed the setup, thus minimizing possible noise due to stray light or other electromagnetic signals, and placed the entire setup with enclosure on an optical table. Data acquisition was performed with different sampling rates resulting in rheological data for different frequency ranges. These were stitched together, thus providing us with a wide frequency response. Local heating of the probing bead due to absorption of the focused laser light was kept minimal by choosing a $975 \mathrm{~nm}$ laser and short acquisition times (several seconds).

We used homebuilt flat sample chamber with $50 \mu \mathrm{L}$ of sample and sealed it with epoxy glue to prevent evaporation. The target concentration of poly(L-lysine)-poly(ethylene glycol) (PLL(20kDa)-g(3.5)-PEG(2kDa)) coated silica colloids (1.5 $\mu \mathrm{m}$ diameter, obtained from Microparticles $\mathrm{GmbH}$, Germany) was typically $10^{-4} \% \mathrm{w}$. Note that the PLL-PEG surface coating prevented possible interactions between the probe and the DNA-network we studied. ${ }^{13}$

\subsection{Dynamic light scattering (DLS)}

DLS MR measurements were performed by a Malvern Zetasizer Nano ZSP (633 nm laser) with temperature control, using $200 \mathrm{~nm}$ azide-functionalized polystyrene particles ( $\mathrm{N}_{3}-\mathrm{PS}$; obtained from Cambridge Bespoke Colloids, UK) as tracer particles. In light-scattering MR, it is paramount that the tracer gives rise to single-scattering events. For this 
reason, the setup was operated in a $173^{\circ}$, non-invasive backscatter (NIBS) detection mode, and the probe concentration used was $0.01 \% \mathrm{w} / \mathrm{v}$ in all measurements. The scattering intensity of the tracers relative to that of the sample was in excess of $95 \%$ in the entire temperature range studied, thus ensuring that the probe scattering dominates the signal. Measurements were done with $40 \mu \mathrm{l}$ disposable cuvettes (Malvern ZEN0040), and the measurement position and attenuator filters were automatically optimized for the best signal acquisition.

The scattering-intensity autocorrelation $g^{(2)}(t)$ is related to the intermediate scattering function $g^{(l)}(t)$ via the Siegert relation:

$$
g^{(2)}(t)=1+\beta\left|g^{(1)}(t)\right|^{2},
$$

where $\beta$ is the zero-time intercept, $g^{(I)}(t)$ is fitted to a multi-exponential decay function via a constrained regularization method (CONTIN) $)^{14}$ using a custom MATLAB code developed in-house. The fit minimized the noise in the experimental curves and smoothened them prior to conversion from real to Fourier space, ensuring high data quality. Furthermore, the generality of the choice of fitting ensured that the data were not pre-fitted to existing models, which could have induced artefacts when converting to Fourier space, as demonstrated elsewhere. ${ }^{15}$ The particle's MSD is related to the intermediate scattering function by:

$$
g^{(1)}(t)=e^{-\frac{q^{2}}{6}<\Delta r^{2}(t)>},
$$

where $q$ is the scattering vector. Finally, the MSD was converted into its Fourier space counterpart and the complex viscoelastic modulus was obtained from the GSER according to the method described before.

\subsection{Video particle tracking (VPT)}

Particle tracking was done on a custom-built setup ${ }^{16,17}$ based on a Nikon Eclipse Ti-E inverted microscope equipped with a 60x 1.2 NA Plan Apo VC objective and a CMOS Point Grey Grasshopper 3 (GS3-U3-23S6M-C, sensor Sony IMX174). We injected the sample into an identical flat chamber as for the OT experiments (preparation described above). Equal mixtures of $420 \mathrm{~nm}$ and $800 \mathrm{~nm}$ azide-functionalized polystyrene ( $\mathrm{N}_{3}$-PS; Cambridge Bespoke Colloids, UK) particles were added to the sample to a final concentration of $10^{-4} \% \mathrm{w}$. Particle MSDs were obtained by averaging over 10 position-tracking acquisitions, each taken for $10 \mathrm{~s}$ with a frame rate of $1 \mathrm{kHz}$. Bright-field microscopy enabled recording cross-correlations in the intensity pattern of the particles' Airy diffraction disks. From these we extracted the particles' positions as a function of time. Matlab routines, developed in-house, were used to convert the generated bead displacements into MSDs that were then transformed into the elastic $G^{\prime}(\omega)$ and viscous $G^{\prime \prime}(\omega)$ moduli.

\subsection{Shearing by conventional bulk rheology (BR)}

Oscillatory shear-measurements were performed in a stress-controlled bulk rheometer (MCR 501, Anton-Paar, Physica) using a cone-and-plate geometry (CP25-2TG, $25 \mathrm{~mm}$ diameter base and $2^{\circ}$ cone angle). Frequency sweeps were run for two different temperatures, $10^{\circ} \mathrm{C}$ and $20^{\circ} \mathrm{C}$. A custom-made vapor trap was used to minimize evaporation effects.

\section{MICRORHEOMETER CALIBRATION AND DATA ANALYSIS}

\subsection{QPD calibration}

Quadrant photodiodes are excellent in detecting the position of the trapped bead within microseconds; however, their continuous analog output requires digitization and calibration. Connecting the diode to an oscilloscope can easily tackle the first problem by digitizing the QPD's analog signals and then forwarding them to the computer, where the analysis takes place. Calibration, on the other hand, is slightly more involved, but can be done in a variety of ways. Here we compare the following: a fully developed self-sustained method (power-spectral density roll-off), which yielded both trap stiffness and position sensitivity; a passive calibration method (equipartition), which provided measure of the trap stiffness; an active calibration method (stuck-bead), which provided measure of the position sensitivity.

Berg-Sørensen and Flyvbjerg ${ }^{18}$ showed how the power-spectral density (PSD) of a trapped particle can be used to extract the trap stiffness and sensitivity of the OT system. Our results, based on their method, are presented in Figure 2. The 
shape of the curves in Figure $2 a$ originated from the fact that the trapped $1.5 \mu \mathrm{m}$ large silica bead showed subdiffusive behavior in deionized water. Imposing a binning procedure that averages the raw data points stored within each bin, we easily fitted a Lorentzian assuming the central limit theorem. The resulting fit allowed converting the voltage and laser driving power (LDP) data into physically meaningful values for the bead displacements and trap stiffness. The higher the value of the crossover frequency $\left(f_{c}\right)$, the stiffer the trap and the less pronounced the diffusion of the bead. An important conceptual point is that the optical trap can be approximated by a parabolic potential for small particle displacements from the trap center, which was located near the focal point of the laser. This allowed drawing an analogy with a harmonic restoring force that pulled the bead back towards the beam waist. Our trap calibration data showed slight anisotropy in trap stiffness, but overall a reasonably good agreement between the PSD roll-off and equipartition method. The latter uses predetermined position sensitivity from the PSD roll-off, which is needed to convert the MSD into units of $\mathrm{m}^{2}$. The equipartition calibration is also based on the assumption that the thermal energy is distributed equally across all degrees of freedom of the bead. Equating this to the harmonic restoring force stored in a single degree of freedom $\left(0.5 k_{B} T=0.5 \kappa<x^{2}\right\rangle$, where $\left\langle x^{2}\right\rangle$ is the MSD in 1D), we obtained the trap stiffness $\kappa$. The general trend of a linear increase of trap stiffness with LDP can be clearly seen in Figure $2 b$.

As a further check, we compared the PSD roll-off results with our stuck-bead calibration data. The latter consisted of the laser scanning a bead stuck to one of the flat chamber surfaces and analyzing the resulting signal. The scaling ratio between voltage signal on the oscilloscope and real bead displacement data was extracted by translating the beam along the particle's surface. Scanning the bead in such a fashion allowed relating the known translational velocity to the timevarying positional signal on the oscilloscope. Piezo drives (precision of $\pm 20 \mathrm{~nm}$ ) allowed precise determination of the actual scanning speed, which was then related to the slope of the graph in Figure $3 a$. The obtained values from the stuckbead method were then compared with the ones from PSD roll-off measurements in Figure $3 b$. In spite of the evident anisotropy in sensitivity, again we concluded there was sufficiently good agreement between the calibration methods.

(a)

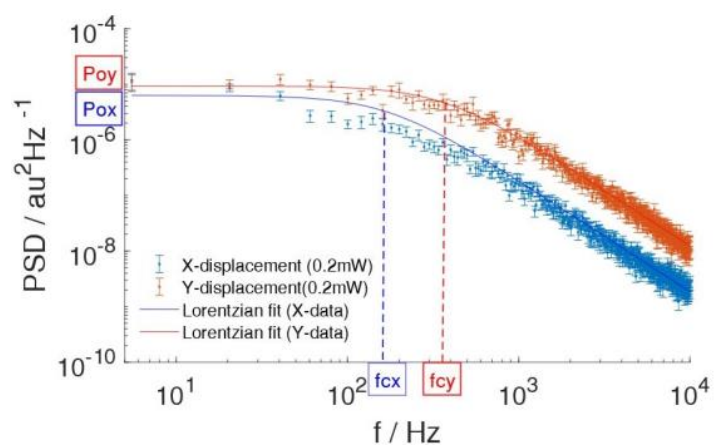

(b)

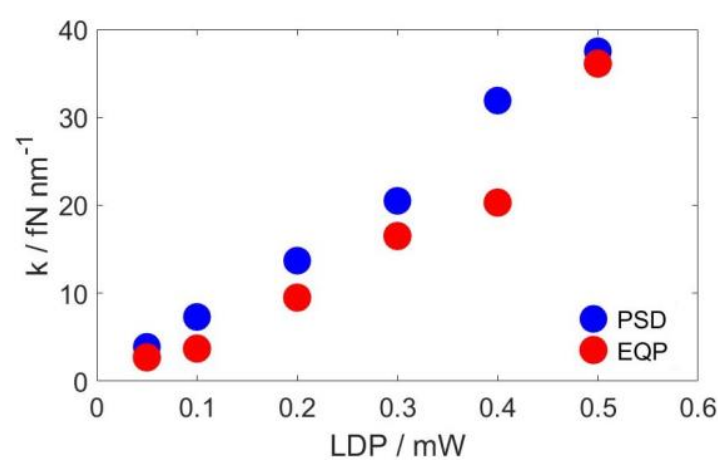

Figure 2. (a) PSD along each lateral displacement direction for a $1.5 \mu \mathrm{m}$ silica bead in deionized water; LDP used was $0.20 \mathrm{~mW}$. The corner frequency $\left(f_{c}\right)$ and the noise power intercept $\left(P_{o}\right)$ provided measures of the trap stiffness and the responsivity (sensitivity) respectively. (b) Comparison between the PSD roll-off and equipartition calibration methods. The former yields both the trap stiffness and responsivity, while equipartition only gives a measure of the trap stiffness.

(a)

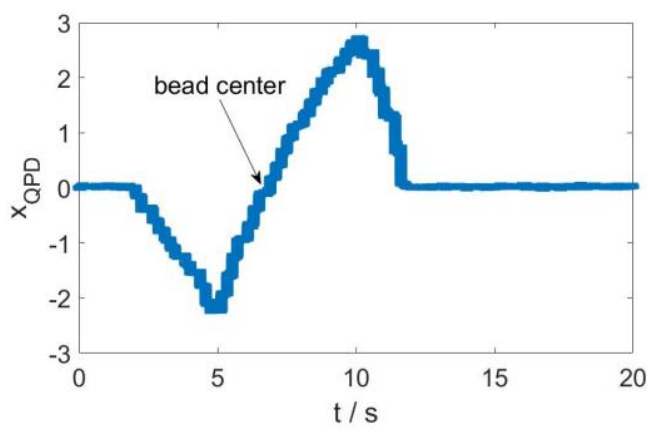

(b)

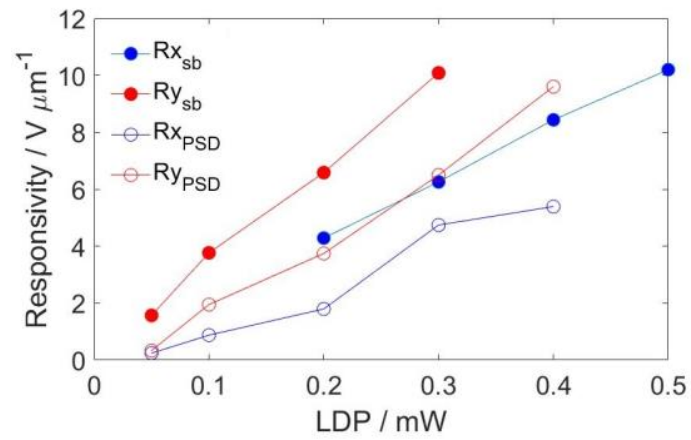

Figure 3. (a) Stuck-bead calibration for using a $1.5 \mu \mathrm{m}$ silica bead in deionized water; the actual scanning speed was $0.1 \mu \mathrm{m} \mathrm{s} \mathrm{s}^{-1}$ and was compared to the slope of the graph. The node of the sinusoid corresponded to scanning the bead center, where the QPD displacement signals cancelled. (b) Comparison between responsivity (sensitivity) results from stuck-bead (filled circles) and PSD roll-off (empty circles) calibration methods; data shown for scanning along the orthogonal transverse directions ( $x$ : blue; $y$ : red). 
Having subjected the PSD roll-off to a test and verified its appropriate use, in the following analysis we solely relied on calibration data obtained from that method. In order to assert the credibility of any subsequent MR measurements, we recalibrated the setup every time we changed the alignment or the sample. Following initial calibration checks, the same values for the LDP and bead positions measured relative to the QPD were used.

\subsection{Microrheology analysis}

Once we acquired the bead's trajectory via our QPD, the complex viscoelastic modulus of the material was calculated according to the GSER relation (Equation (1)). The latter required performing a Fourier transform of the MSD. It can be shown that in the case of a bound particle, the position-autocorrelation function (PACF) contains the same information as the MSD and could be used in its place. ${ }^{19}$ The raw PACF, however, becomes rather noisy at long lag times due to poor statistics. This severely affects the quality of the resulting numerical Fourier transform. Tassieri et al. ${ }^{19}$ offered a solution to this problem by suggesting a logarithmic binning procedure, which effectively reduces the sampling density at long lag times and significantly lowers the noise. Applying this method to the PACF of a $1.5 \mu \mathrm{m}$ silica bead, trapped in deionized water, resulted in the graph shown in Figure $4 a$. The logarithmically binned PACF was then converted via Fourier transform into storage and loss moduli, based on the scheme outlined by Evans et al. ${ }^{8}$ (Figure 4b). Trapping the bead with the laser introduced an additional apparent elasticity $\left(G_{\text {eff }}^{\prime}=G_{\text {true }}^{\prime}+\kappa /(6 \pi a)\right)$. The actual viscoelastic modulus was then obtained by linearly subtracting the additional elastic trap contribution. This resulted in a negligible storage modulus for deionized water. Furthermore, the loss modulus showed a linear frequency dependence, as expected for a Newtonian liquid.

(a)

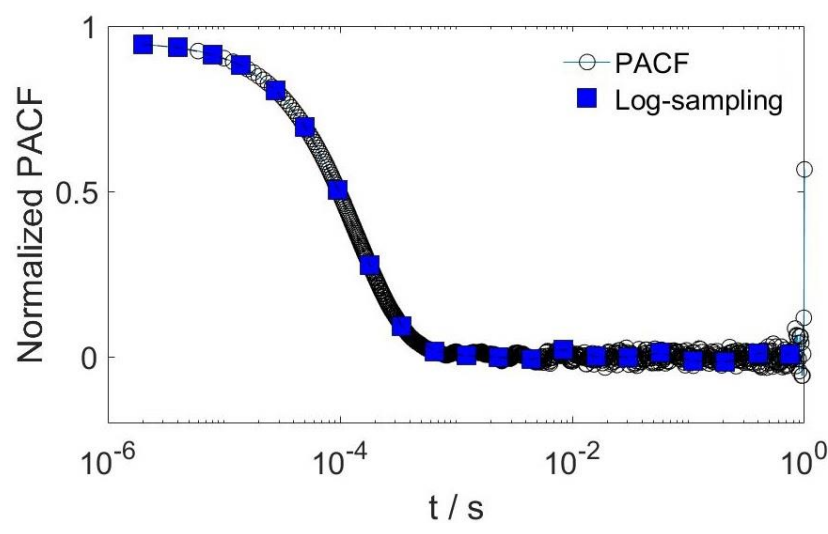

(b)

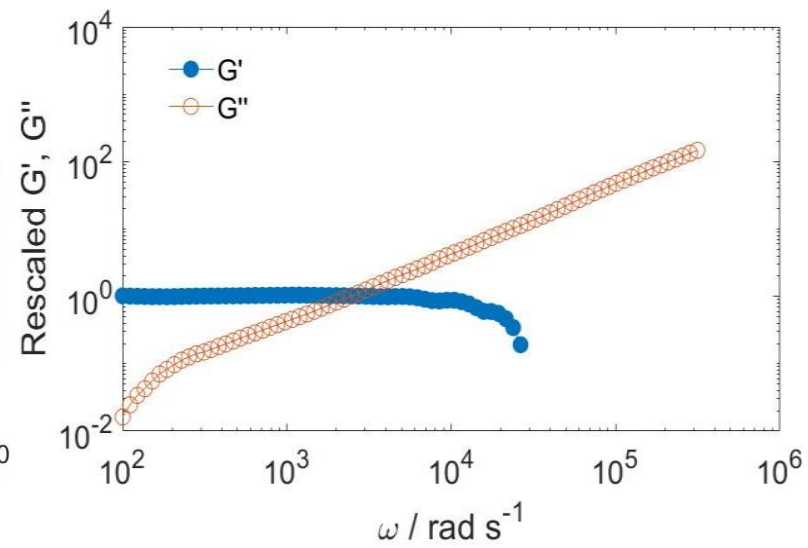

Figure 4. (a) Normalized PACF of an optically trapped $1.5 \mu \mathrm{m}$ silica bead in deionized water $(0.5 \mathrm{~mW}$ LDP). The raw dataset was sampled logarithmically to yield a representative set of 100 points (blue squares), which was then Fourier transformed to obtain viscoelasticity of the medium in frequency space. (b) The storage ( $G^{\prime}$, filled circles) and loss ( $G^{\prime}$,', open circles) moduli plotted against angular frequency and rescaled by $\kappa /(6 \pi a)$. The apparent elasticity (plateau region) comes from the trap stiffness $\kappa$ and depends linearly on the laser power.

\section{SAMPLE CHARACTERIZATION AND RHEOLOGY}

\subsection{Sample characterization}

The DNA hydrogel samples were prepared by first combining equal concentrations of single strands SS 1-3 to form the Y-shapes, and separately SS 4-6 to form the Y'-shapes, and then cooling the samples slowly from $80^{\circ} \mathrm{C}$ to $30^{\circ} \mathrm{C}$ to ascertain equilibrium hybridization (binding). Once formed, the $\mathrm{Y}$ - and $\mathrm{Y}^{\prime}$-shaped nanostars were mixed at equal proportions in $1 \mathrm{x}$ TE buffer at $\mathrm{pH} 8$, containing $\sim 200 \mathrm{mM} \mathrm{NaCl}$ at room temperature. The thermodynamics of the equilibrium melting and hybridization behavior of ds DNA is well-known. The binding energy is determined by the number of hydrogen bonds that can form between the only possible pairing between the adenine and thymine bases (AT) and the guanine and cytosine bases (GC), where AT forms two and GC - three hydrogen bonds. These break at high temperatures and reform at lower $T$. Hence, the formation of the DNA nanostars is thermally reversible. In order to make 
sure that these Y-shaped DNA nanostars remained inert between $20^{\circ} \mathrm{C}$ and $30^{\circ} \mathrm{C}$, we designed and tested their melting behavior using UV-vis. spectroscopy, making use of the fact that ds DNA absorbs the wavelength of $260 \mathrm{~nm}$ less strong than ss DNA. We present these melting curves for the formation of the Y-and Y'-shapes in Figure 5a. The typical Sshaped curves show for both nanostars an almost identical melting temperature $T_{m} \approx 64-65^{\circ} \mathrm{C}$, which is the temperature, at which half of all possible hydrogen bonds between the SS DNA strands have formed. ${ }^{12}$ We further see that all nanostars have fully formed below about $55^{\circ} \mathrm{C}$.

As we wanted to investigate the viscoelastic properties of our hydrogels as they formed through binding between the sticky ends of the nanostars, we chose the length and binding strength between the complementary sticky ends to correspond to a melting temperature of around $20^{\circ} \mathrm{C}$. In Figure $5 b$, we show the UV-vis. melting curves obtained for the isolated sticky ends. The curves indeed reflect the theoretically predicted curves based on the SantaLucia model. ${ }^{12}$ They also confirm that when we melt the system to about $30^{\circ} \mathrm{C}$, it is a completely fluid suspension of $\mathrm{Y}$-shaped building blocks, but then starts to form a viscoelastic network as we cool the system down to $10^{\circ} \mathrm{C}$. This gelation behavior is fully thermally reversible.

(a)

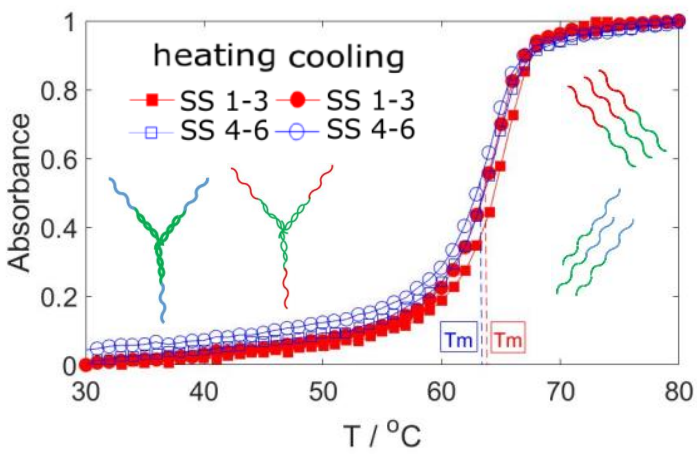

(b)

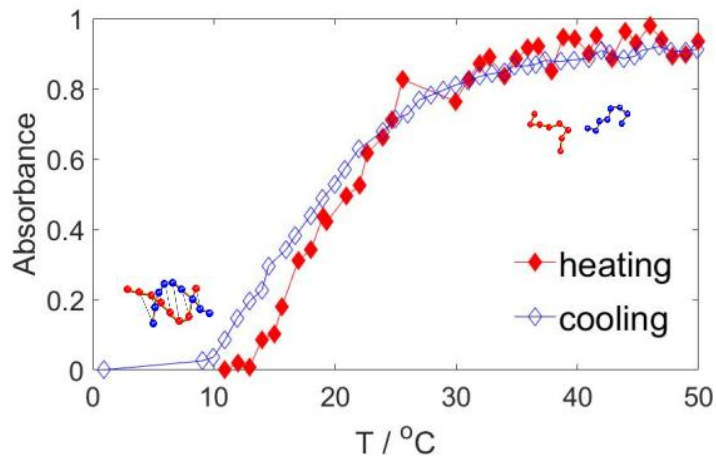

Figure 5. (a) Normalized UV-vis. melting curves of the two nanostars formed by single DNA strands SS 1-3 (blue sticky ends) and SS 4-6 (red sticky ends), revealing a similar melting temperature $T_{m} \approx 64-65^{\circ} \mathrm{C}$. Here the circles indicate measurements taken for a cooling cycle, and the squares - for a heating cycle. (b) Normalized UV-vis. melting curves measured for the sticky ends only, not containing the ds core of the nanostars.

\subsection{Rheology of DNA hydrogels}

This section aims at presenting the rheology results for the gelation process of DNA-nanostars obtained from different experimental techniques: passive MR using single-beam OT, DLS, VPT and BR. By choosing these four methods, we were able to compare and complement the extracted high-frequency viscoelasticity (OT and DLS) with the lowfrequency sample behavior (VPT and BR). The samples were prepared as outlined in Section 2 and the summarized results from the rheology are presented in Figures 6 and 7.

In Figure $6 a$ we show the normalized PACF obtained from OT measurements and the extracted viscoelastic moduli $G^{\prime}(\omega)$ and $G^{\prime \prime}(\omega)$. The temperature dependence of the decay in the PACF curves and the moduli clearly demonstrates that the system transforms from a fluid of Y-shapes into an increasingly more elastic gel as the sample is cooled from above the $T_{m}$ of the sticky ends to below that temperature. At $15^{\circ} \mathrm{C}$, the moduli ran almost parallel, showing approximately identical scaling of $\omega^{0.5}$. Such a scaling behavior was identified by Winter and Chambon ${ }^{20}$ as the point, at which an initially fluid polymer solution becomes a percolating, crosslinked network, and which is a synonym of the gelpoint of the system. We observed such a behavior as well in DNA hydrogels formed by similar Y-shaped DNA building blocks ${ }^{12}$ and could identify this gelpoint with the $T_{m}$ of the system's sticky ends. It is important to note that slightly above $T_{m}$, the system turns into a cluster fluid, while below $T_{m}$, the system becomes increasingly more elastic. However, once all possible bonds between the $\mathrm{Y}$ - and $\mathrm{Y}^{\prime}$-shapes have formed (below $10^{\circ} \mathrm{C}$ ), the elasticity of the system did no longer increase.

It is important to note that the viscoelasticity measurements with the OT are accompanied by significant limitations to the duration and power of the laser used in each individual acquisition. By varying the LDP, we found that at high trapping power the decay of the PACF reflected how quickly the bead was dragged back towards the focal point and contained very little information about the internal structure of the sample. Therefore, it was necessary to use sufficiently 
low LDP in order to observe a PACF decay that is truly governed by the stiffness of the gel. Additionally, long exposure of the sample to the laser is known to cause local heating that in some cases could lead to the melting of the DNA bonds in the gel. This posed a firm threshold both to the used LDP and the duration of an acquisition. Due to all of the above, the presented results reflect data acquired at $100 \mathrm{kHz}$ for a period of $10 \mathrm{~s}$ at LDP of $0.10 \mathrm{~mW}$. The low-frequency noise observed at $25^{\circ} \mathrm{C}$ was a consequence of the short measurement duration, where the dataset suffered from poor statistics. Below $15^{\circ} \mathrm{C}$, trapping the bead with LDP of $0.10 \mathrm{~mW}$ became increasingly more challenging and the signal-to-noise ratio gradually decreased.

(a)
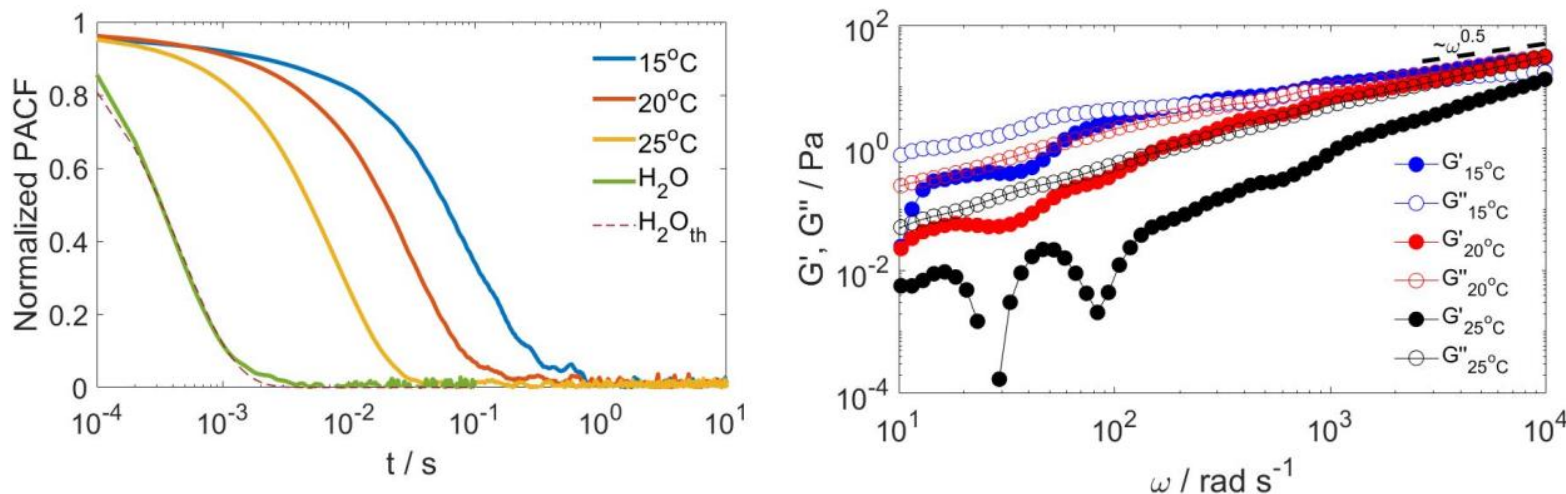

(b)
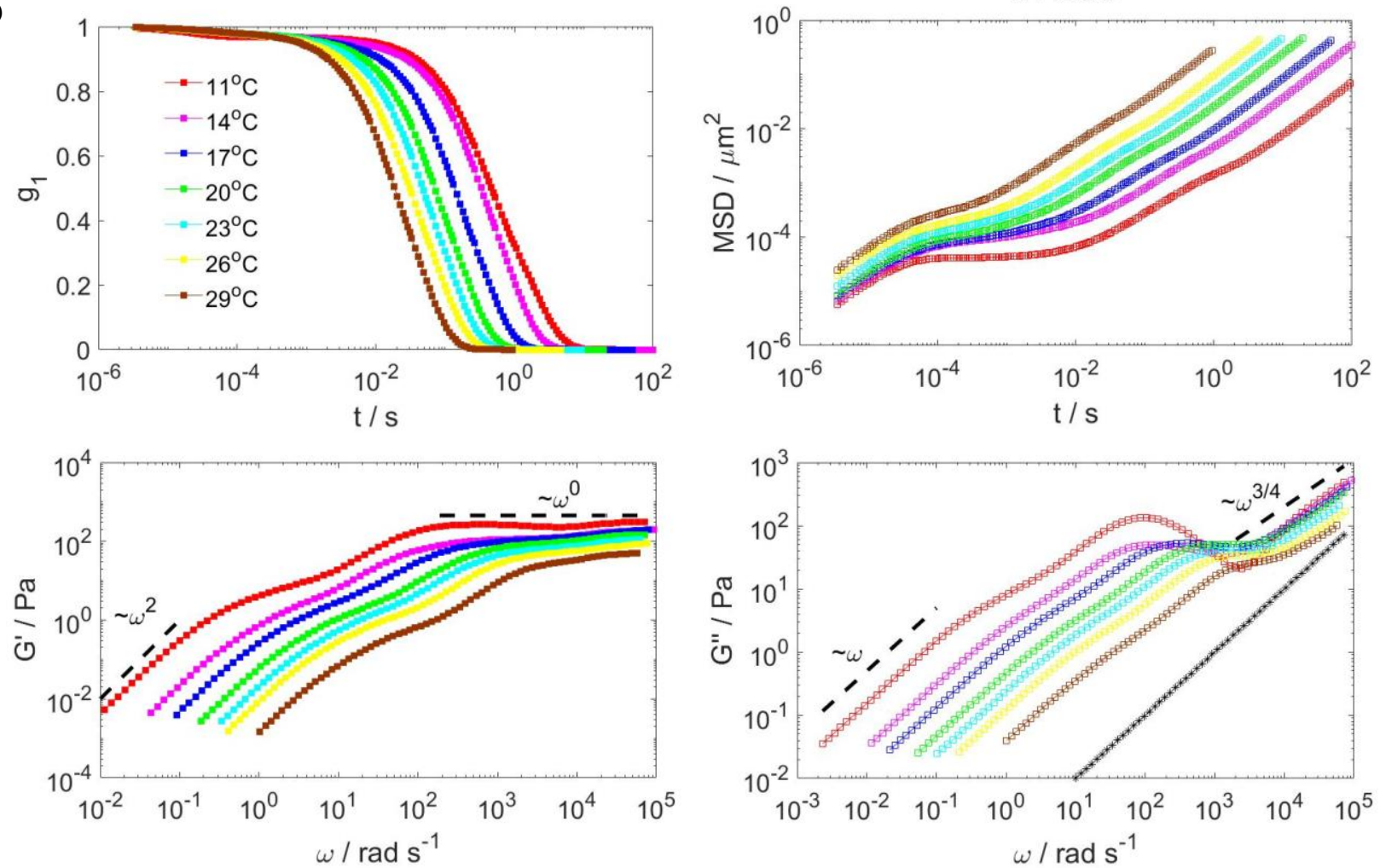

Figure 6. (a) OT measurements: normalized PACF and the corresponding storage (filled) and loss moduli (open circles) at $15^{\circ} \mathrm{C}$, $20^{\circ} \mathrm{C}$ and $25^{\circ} \mathrm{C}$; experimental (green) and theoretical (dashed) curves for water. (b) DLS measurements: intensity-autocorrelation function $\mathrm{g}_{1}$ and MSD with the corresponding storage (filled) and loss moduli (open squares) at $11-29^{\circ} \mathrm{C}$; water at $20^{\circ} \mathrm{C}$ (stars).

An alternative dataset, provided by DLS measurements, is shown in Figure $6 b$. The intensity-autocorrelation function was found to behave similarly to the PACF in the trapping experiments, with the decay becoming longer at lower temperatures. The increasingly constrained phase space that the bead was allowed to explore on cooling was evident 
from the subdiffusive motion in the MSD curves at intermediate times. Following the trend in $G^{\prime}(\omega)$ and $G^{\prime \prime}(\omega)$ from $30^{\circ} \mathrm{C}$ down to $11^{\circ} \mathrm{C}$, one could directly compare the viscoelastic behavior of the gel with the one predicted by Grimm et $a l .^{21}$ for a Maxwell fluid with a single-relaxation time.

The Maxwell model represents the viscous and elastic behavior of a sample by modelling the mechanical response of a viscous dashpot and an elastic spring connected in series. We found that the measured $G^{\prime}(\omega)$ and $G^{\prime \prime}(\omega)$ display similar scaling laws as those of a Maxwell fluid, with a characteristic dip in $G^{\prime \prime}(\omega)$ at intermediate frequencies. This behavior was caused by the presence of the background solvent. At low angular frequencies, the linear scaling of the loss modulus provided a measure of the zero-shear viscosity of the sample. The storage modulus, on the other hand, increased quadratically with frequency, in agreement with the model and described in detail elsewhere. ${ }^{22}$ In the intermediate frequency range, there was a predominantly elastic response. The first crossing point of the two moduli provided information about the longest relaxation time of the hydrogel $(\sim 50 \mathrm{~ms})$, and was found to be comparable to other trivalent DNA systems we have investigated earlier. ${ }^{12}$

At frequencies above $100 \mathrm{rad} \mathrm{s}^{-1}$, the storage modulus reached a plateau at around $250 \mathrm{~Pa}$, which was expected to grow in magnitude and extend over a larger range for lower temperatures. This plateau value could be used in conjunction with the thermal energy to provide an estimate of the average mesh size $\xi \sim\left(k_{B} T / G^{\prime}\right)^{1 / 3} \approx 25 \mathrm{~nm}$ present in the network formed at $11{ }^{\circ} \mathrm{C}$. This corresponds to approximately the average distance between the centers of two bonded Y-shapes. For tetravalent DNA systems it was predicted that the constant low-temperature limiting value of $G^{\prime}(\omega)$ is approximately 600 $\mathrm{Pa}^{23}$ If we assume each arm of the nanostar to contribute $150 \mathrm{~Pa}$, then we expect a $G^{\prime}(\omega)$ terminal value of $450 \mathrm{~Pa}$ for our system as the temperature is decreased even further. At very high frequencies $\left(\geq 10^{4} \mathrm{rad} \mathrm{s}^{-1}\right)$, the $G^{\prime \prime}(\omega)$ scaled as $\omega^{3 / 4}$, which is known from theory to correspond to semi-flexible polymer networks. We believe this to be a general feature of crosslinked DNA gels, which remains yet to be confirmed through investigating other systems.

In an attempt to further explore the resemblance of the DNA high-frequency behavior to that of a semi-flexible polymer, we followed the theoretical analysis of David Morse. ${ }^{24}$ We were particularly interested in estimating (based on our rheological data) the DNA persistence length, i.e. roughly the distance, over which the DNA chain could be considered rigid. Using the high-frequency slope of the loss modulus $\left(G^{\prime \prime}(\omega) \approx 2^{0.75} \rho\left(k_{B} T\right)^{0.25} \xi^{0.75} L^{1.25} \omega^{0.75} / 15\right.$ with $\rho$ being concentration of contour length per unit volume and $L$ is the persistence length), we calculated a persistence length of 45 $\mathrm{nm}$ at $11^{\circ} \mathrm{C}$ for the mesh size determined previously. This compares favorably with other results reported in literature, ${ }^{25}$ where the slightly lower value could be attributed to the presence of a flexible thymine linker.

In order to confirm the measured low-frequency viscoelasticity of the DNA hydrogel, we also conducted MR by VPT and conventional BR. Video recordings via bright-field microscopy allowed tracking the trajectory of a freely diffusing bead through the same DNA hydrogel and thus provided a measure of the bead's MSD (Figure 7a). Due to the fact that we used beads of two different sizes, we rescaled the MSD by the bead diameter in each case. At high temperatures (20$26^{\circ} \mathrm{C}$ ), we followed the motion of $800 \mathrm{~nm} \mathrm{~N}$-PS particles. At $17^{\circ} \mathrm{C}$, we made an equal number of acquisitions with 800 $\mathrm{nm}$ and $420 \mathrm{~nm} \mathrm{~N}$-PS particles and hence we scaled the MSDs by the appropriate factors to reflect the change in diffusivity. At $11-14^{\circ} \mathrm{C}$, we followed the motion of $420 \mathrm{~nm} \mathrm{~N}$-PS particles. The overall trend was again as expected and in complete agreement with our previous measurements. In spite of the presence of some low-frequency noise, we concluded that as the temperature increased, the crossover between $G^{\prime}(\omega)$ and $G^{\prime \prime}(\omega)$ occurred at higher values of $\omega$. This suggests that the stiffening of the DNA hydrogel corresponds to a longer relaxation time of the percolating network.

BR measurements, summarized in Figure $7 b$, did not provide any conclusive evidence for a particular trend. On the contrary, we suspected possible artefacts appearing at high frequency due to the inertia of the instrument and insufficient time allowed for collecting the data. Whereas small geometries, such as the $25 \mathrm{~mm}$ cone-and-plate are often used with costly samples, such as DNA hydrogels, they are much more susceptible to failure due to edge effects and evaporation. In spite of our efforts to minimize these errors by careful sample loading and the use of a vapor trap, we could not completely overrule any possible deviation arising from those unwanted side effects.

Finally, we conclude by providing a comparison between the results shown above. Reporting broadband viscoelasticity is not uncommon, with some good examples in the literature of how this is best done through MR. ${ }^{26,27,28}$ Here, we aimed at comparing different techniques, emphasizing the suitability of using optical trapping with the setup shown in Section 2. OT have some clear advantages over other setups in that they provide local information about small volumes of expensive or scarce samples in a non-invasive fashion. However, as already discussed, in MR laser tweezers have to be used with care by considering the details of how to conduct a measurement in a way that avoids artefacts. This includes testing different laser powers, sampling rates, acquisition durations and particle-probes. Combining acquisitions of 
different length and sampling rate could in principle yield viscoelastic data in the range $10^{1}-10^{6} \mathrm{~Hz}$. Moreover, stitching these signals to data from BR or VPT could extend the range over 10 orders of magnitude in frequency. Due to the complicated nature of DNA hydrogels, we expected to obtain viscoelasticity over a more limited range. The merging of different sets of viscoelastic data is demonstrated in Figure 8.

Figure 8a shows an attempt to match the high-frequency viscoelasticity measured by OT to the low-frequency BR data. Continuous QPD signals were sampled by the oscilloscope with two different rates $-1 \mathrm{MHz}$ and $100 \mathrm{kHz}$. We observed the appearance of noise near the terminal points in each dataset; hence the significance of stitching results together to produce one coherent graph with few artefacts becomes apparent. For instance, instrumental inertia effects are known to exacerbate the data quality in BR measurements as the frequency is increased (50-100 rad s $\mathrm{s}^{-1}$. Taken as an individual result, it might be interpreted as suggesting a change in the scaling behavior of the storage and loss moduli. To verify the collected BR data, we successfully match the results to viscoelasticity obtained with OT at $20^{\circ} \mathrm{C}$.

(a)
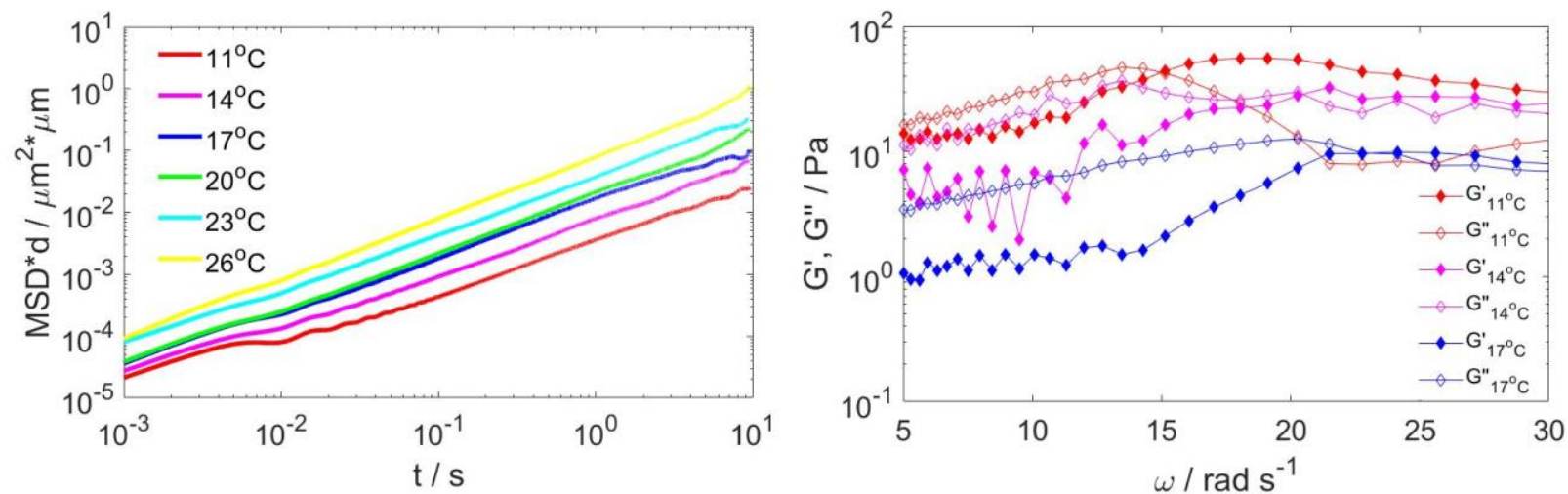

(b)

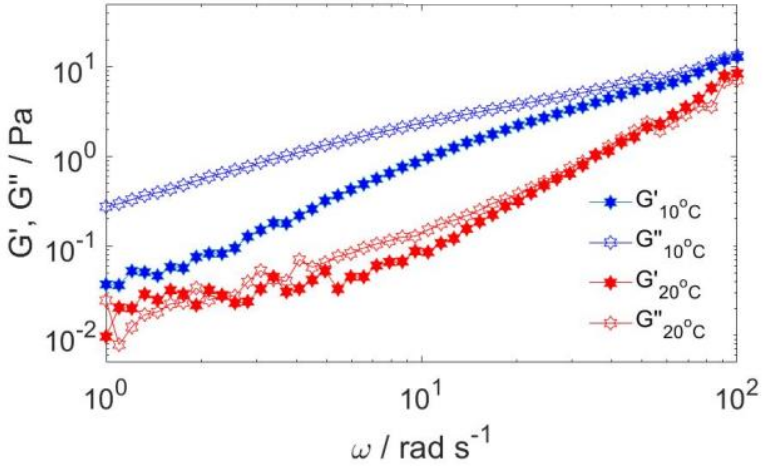

Figure 7. (a) VPT measurements: MSD scaled by the bead diameter and the corresponding storage (filled diamonds) and loss moduli (open diamonds) for $11-17^{\circ} \mathrm{C}$. (b) BR measurements: storage (filled hexagons) and loss moduli (open hexagons) obtained from frequency sweeps at $10^{\circ} \mathrm{C}$ and $20^{\circ} \mathrm{C}$; the geometry used in the oscillatory measurements was 25 mm diameter cone-andplate.

A comparison between data extracted from OT, DLS and VPT at $25^{\circ} \mathrm{C}$ provided yet another sanity check (Figure $8 b$ ). In spite of the general good agreement, we want to emphasize the large scatter of data points at low frequency using optical trapping. As previously mentioned, the analysis we used here required subtracting the elastic contribution from the focused laser light trapping the bead. After removing this additional contribution, we were left with elasticity, which was rather low at temperatures above melting. Due to averaging over insufficient number of points separated by a long enough time, the estimated errors were too large on the scale of the absolute magnitude of the moduli. In addition, as reported by Gardel et al. ${ }^{29}$ the appearance of compressional modes is known to prevent obtaining reliable low-frequency data using MR since their decoupling is highly non-trivial. These problems were nearly absent in DLS, though it is the ensemble-averaged response that was obtained. Artefacts appeared also in the VPT data, where we experienced problems with mechanical and electrical noise. 
The inverse temperature dependence of the infinite-time viscosity is evident from Figure $8 d$. This behavior is common for many pure liquids, and is also a property of our system here. The theoretical curve, corresponding to the steady-state viscosity of water at $20^{\circ} \mathrm{C}$, is also shown as a reference (black star symbols). All curves, displayed in Figure $8 d$, showed the sample in its liquid form, where the additional viscosity originated from the individual DNA clusters formed in the bulk. Figure $8 \mathrm{c}$ presents the gel state of the sample at $11^{\circ} \mathrm{C}$, where the elastic modulus dominated over the viscous modulus. Again, at the gel point, the real and imaginary parts of the complex viscoelastic modulus were expected to run approximately parallel to one another, in agreement with the findings of Winter and Chambon. ${ }^{20}$ Comparing these results with those presented in Figure 6, we conclude that the melting transition of the DNA hydrogel is bound to occur at around $14^{\circ} \mathrm{C}$.

(a)

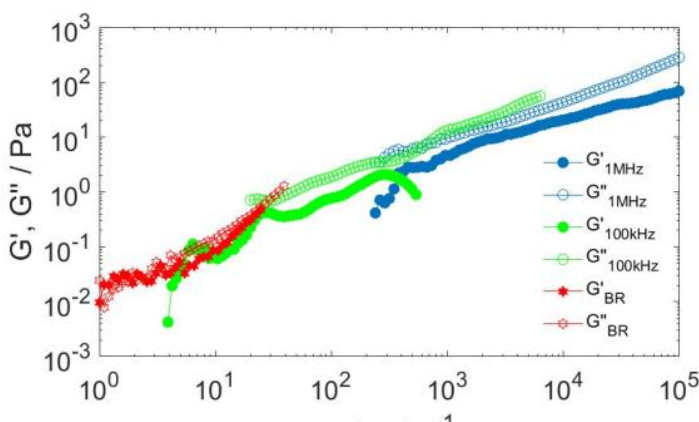

(c)

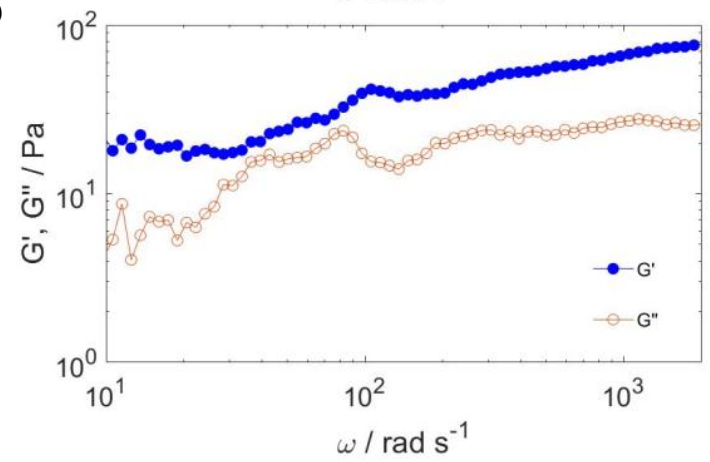

(b)

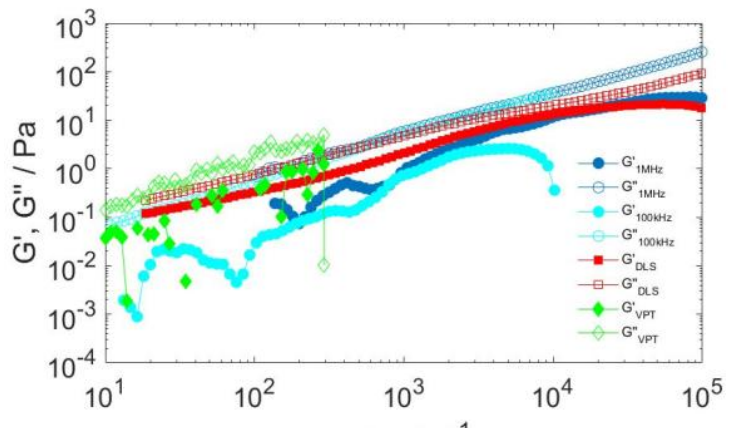

(d)

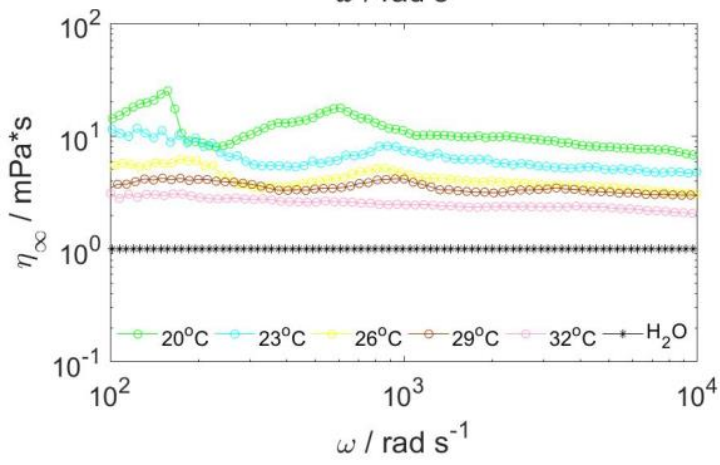

Figure 8. Comparison between rheology results obtained from different experimental techniques: (a) OT measurements (sampling rates of $1 \mathrm{MHz}$ in blue and $100 \mathrm{kHz}$ in green) and $\mathrm{BR}$ measurements (red) at $20^{\circ} \mathrm{C}$; (b) OT measurements $(1 \mathrm{MHz}$ in dark blue and $100 \mathrm{kHz}$ in light blue), DLS measurements in red, and VPT measurements in green $\left(\mathrm{T}=25^{\circ} \mathrm{C}\right)$; (c) OT measurements: gelling of the hydrogel found at $11^{\circ} \mathrm{C}(100 \mathrm{kHz}$ sampling rate); (d) OT measurements: DNA hydrogel infinite-time viscosity at different temperatures $\left(1 \mathrm{MHz}\right.$ sampling rate) - black stars denote water viscosity at $20^{\circ} \mathrm{C}$. Filled symbols represent storage moduli and empty symbols represent loss moduli in (a)-(c).

\section{CONCLUSIONS}

Passive MR allows investigating the mechanical properties of complex fluids on different timescales. After careful calibration and testing, an OT system could be used to complement other experimental techniques, such as DLS, VPT and conventional BR. Investigating the scaling of the storage and loss moduli can provide information about the melting temperature and mesh size of a DNA hydrogel. While the viscoelasticity of our nanostars followed the single-relaxation Maxwell fluid model at low and intermediate frequencies, at high frequencies we observed the signature of a semiflexible polymer network. This insight allowed us to calculate the mechanical properties of DNA, such as its persistence length, which was found to agree with accepted values.

\section{ACKNOWLEDGEMENTS}

We thank Dr Jurij Kotar for useful discussions related to the build-up and calibration of the optical tweezers. I. D. S. acknowledges financial support from EPSRC. E. E. and A. C. thank ETN-COLLDENSE (H2020-MCSA-ITN-2014, 
grant no. 642774). Z. X. acknowledges support from National University of Defense Technology Scholarship and Jesus College, the University of Cambridge. E. E. also thanks the Winton Program for Sustainable Physics.

\section{REFERENCES}

[1] Ashkin, A., Dziedzic, J. M., Bjorkholm, J. E. and Chu, S., "Observation of a single-beam gradient force optical trap for dielectric particles," Opt. Lett. 11, 288-290 (1986).

[2] Block, S. M., Goldstein, L. S. B. and Schnapp, B. J., "Bead movement by single kinesin molecules studied with optical tweezers," Nature 348, 348-352 (1990).

[3] Wang, M. D., Yin, H., Landick, R., Gelles, J. and Block, S. M., "Stretching DNA with optical tweezers," Biophys. J. 72, 13351346 (1997).

[4] Gibson, G. M., Leach, J., Keen, S., Wright, A. J. and Padgett, M. J., "Measuring the accuracy of particle position and force in optical tweezers using high-speed video microscopy," Opt. Express 16, 14561-14570 (2008).

[5] Gittes, F. and Schmidt, C. F., "Interference model for back-focal-plane displacement detection in optical tweezers," Opt. Lett. 23, 7-9 (1998).

[6] Keen, S., Leach, J., Gibson, G. and Padgett, M. J., "Comparison of a high-speed camera and a quadrant detector for measuring displacements in optical tweezers," J. Opt. Pure Appl. Opt. 9, S264 (2007).

[7] Mason, T. G., "Estimating the viscoelastic moduli of complex fluids using the generalized Stokes-Einstein equation," Rheol. Acto 39, 371-378 (2000).

[8] Evans, R. M. L., Tassieri, M., Auhl, D. and Waigh, T. A., "Direct conversion of rheological compliance measurements into storage and loss moduli," Phys. Rev. E 80, 012501 (2009).

[9] Buchanan, M., Atakhorrami, M., Palierne, J. F. and Schmidt, C. F., "Comparing Macrorheology and One- and Two-Point Microrheology in Wormlike Micelle Solutions," Macromolecules 38, 8840-8844 (2005).

[10] Vermeulen, K. C. and van Mameren, J., "Calibrating bead displacements in optical tweezers using acousto-optic deflectors," Rev. Sci. Instrum. 77, 013704 (2006).

[11] Padgett, M. and Bowman, R., "Tweezers with a twist," Nat. Photonics 5, 343-348 (2011).

[12] Xing, Z., Caciagli, A., Cao, T., Stoev I., Zupkauskas, M., O'Neill, T., Wenzel, T., Lamboll, R., Liu, D. and Eiser, E., "Microrheology of DNA Hydrogels," PNAS (2018).

[13] Yanagishima, T., Di Michele, L., Kotar, J. and Eiser, E., "Diffusive behaviour of PLL-PEG coated colloids on $\lambda$-DNA brushes tuning hydrophobicity," Soft Matter, 8, 6792-6798 (2012).

[14] Provencher, S. W., "A constrained regularization method for inverting data represented by linear algebraic or integral equations," Comput. Phys. Commun. 27, 213-227 (1982).

[15] Maier, T., Boehm, H. and Haraszti, T., "Splinelike interpolation in particle tracking microrheology," Phys. Rev. E 86, 011501 (2012).

[16] Yanagishima, T., Frenkel, D., Kotar, J. and Eiser, E., "Real-time monitoring of complex moduli from micro-rheology," J. Phys. Condens. Matter 23, 194118 (2011).

[17] Di Michele, L., Yanagishima, T., Brewer, A. R., Kotar, J., Eiser, E., Fraden S., "Interactions between Colloids Induced by a Soft Cross-Linked Polymer Substrate," Phys. Rev. Lett. 107, 136101 (2011).

[18] Berg-Sørensen, K. and Flyvbjerg, H., "Power spectrum analysis for optical tweezers," Rev. Sci. Instrum. 75, 594-612 (2004).

[19] Tassieri, M., Evans, R. M. L., Warren, R. L., Bailey, N. J. and Cooper, J. M., "Microrheology with optical tweezers: data analysis," New J. Phys. 14, 115032 (2012).

[20] Winter, H. H. and Chambon, F., "Analysis of Linear Viscoelasticity of a Crosslinking Polymer at the Gel Point," J. Rheol. 30, 367-382 (1986).

[21] Grimm, M., Jeney, S. and Franosch, T., "Brownian motion in a Maxwell fluid," Soft Matter 7, 2076-2084 (2011).

[22] van Zanten, J. H. and Rufener, K. P., "Brownian motion in a single relaxation time Maxwell fluid," Phys. Rev. E 62, 5389-5396 (2000).

[23] Fernandez-Castanon, J., Bianchi, S., Saglimbeni, F., Leonardo, R. D. and Sciortino, F., "Microrheology of DNA hydrogel gelling and melting on cooling," Soft Matter (2018).

[24] Morse, D. C., "Viscoelasticity of Concentrated Isotropic Solutions of Semiflexible Polymers. 2. Linear Response," Macromolecules 31, 7044-7067 (1998).

[25] Marko, J. F. and Siggia, E. D., "Stretching DNA," Macromolecules 28, 8759-8770 (1995).

[26] Tassieri, M., Gibson, G. M., Evans, R. M. L., Yao, A. M., Warren, R., Padgett, M. J. and Cooper, J. M., "Measuring storage and loss moduli using optical tweezers: Broadband microrheology," Phys. Rev. E 81, 026308 (2010).

[27] Preece, D., Warren, R., Tassieri, M., Evans, R. M. L., Gibson, G. M., Padgett, M. J., Cooper, J. M., "Optical tweezers: wideband microrheology," J. Opt. 13, 044022 (2011).

[28] Del Giudice, F., Tassieri, M., Oelschlaeger, C. and Shen, A. Q., "When Microrheology, Bulk Rheology, and Microfluidics Meet: Broadband Rheology of Hydroxyethyl Cellulose Water Solutions," Macromolecules 50, 2951-2963 (2017).

[29] Gardel, M. L., Valentine, M. T. and Weitz, D. A., "Microrheology," Microscale Diagnostic Techniques 1-49 (2005). 